Air-Stable Ir(P-Phos) Complex for Highly Enantioselective Hydrogenation of Quinolines and their Immobilization in Poly(ethylene glycol) Dimethyl Ether (DMPEG)

Chem. Commun. 2005, 1390-1392.

\title{
Immobilized Ir-(P-Phos) Complex for Enantioselective Hydrogenation of Quinolines
}

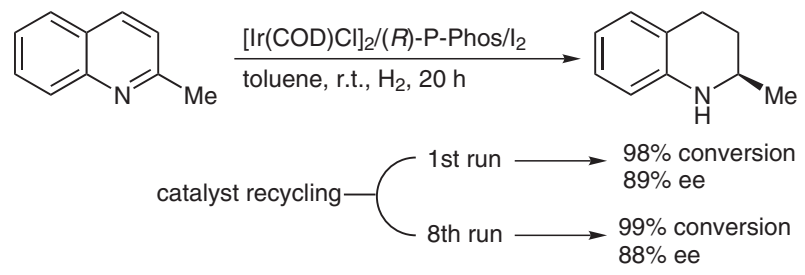

Significance: An air-stable, recyclable Ir-(P-Phos) complex immobilized on poly(ethylene glycol) dimethyl ether (DMPEG) has been established for the hydrogenation of quinolines. The product can be separated by simple extraction with hexanes while the catalyst was retained in the DMPEG phase.
Comment: Reusability and air-stability are desirable features for a hydrogenation catalyst especially for practical applications. These authors have developed a nice immobilized catalyst for asymmetric hydrogenation giving important chiral tetrahydroquinoline-derived products. Enantioselective hydrogenation of quinoline derivatives is still one of the important issues for natural product synthesis. Importantly, the present method can be used for other enantioselective hydrogenations and the reaction conditions tolerate free alcoholic moieties on the substrate as well. 